University of Nebraska - Lincoln

DigitalCommons@University of Nebraska - Lincoln

Faculty Publications from the Department of Engineering Mechanics

2010

\title{
Evaluation of biological cell properties using dynamic indentation measurement
}

\author{
Guoxin Cao \\ University of Nebraska - Lincoln, gcao2@unl.edu \\ Namas Chandra \\ University of Nebraska - Lincoln, nchandra2@unl.edu
}

Follow this and additional works at: https://digitalcommons.unl.edu/engineeringmechanicsfacpub

Part of the Mechanical Engineering Commons

Cao, Guoxin and Chandra, Namas, "Evaluation of biological cell properties using dynamic indentation measurement" (2010). Faculty Publications from the Department of Engineering Mechanics. 72. https://digitalcommons.unl.edu/engineeringmechanicsfacpub/72

This Article is brought to you for free and open access by the Mechanical \& Materials Engineering, Department of at DigitalCommons@University of Nebraska - Lincoln. It has been accepted for inclusion in Faculty Publications from the Department of Engineering Mechanics by an authorized administrator of DigitalCommons@University of Nebraska - Lincoln. 


\title{
Evaluation of biological cell properties using dynamic indentation measurement
}

\author{
Guoxin $\mathrm{Cao}^{*}$ and Namas Chandra ${ }^{\dagger}$ \\ Department of Engineering Mechanics, University of Nebraska-Lincoln, Lincoln, Nebraska 68588-0642, USA \\ (Received 18 May 2009; revised manuscript received 17 September 2009; published 26 February 2010)
}

\begin{abstract}
Viscoelastic mechanical properties of biological cells are commonly measured using atomic force microscope (AFM) dynamic indentation with spherical tips. A semiempirical analysis based on numerical simulation is built to determine the cell mechanical properties. It is shown that the existing analysis cannot reflect the accurate values of cell elastic/dynamic modulus due to the effects of substrate, indenter tip size, and cell size. Among these factors, substrate not only increases the true contact radius but also interferes the indentation stress field, which can cause the overestimation of cell moduli. Typically, the substrate effect is much stronger than the other two influences in cell indentation; and, thus, the cell modulii are usually overestimated. It is estimated that the moduli can be overestimated by as high as over $200 \%$ using the existing analysis. In order to obtain the accurate properties of cells, correction factors that account for these effects are required in the existing analysis.
\end{abstract}

DOI: 10.1103/PhysRevE.81.021924

PACS number(s): $87.10 . \mathrm{Kn}$

\section{INTRODUCTION}

There is a close relationship between the physical functions of cells and their mechanical behavior. For example, Cross et al. recently reported that the stiffness of metastatic cancer cells is $70 \%$ lower than that of healthy cells [1]; the heart muscle cells loss their contractility will cause the heart failure [2]; the traumatic brain injury (TBI) might be caused by the neuron death under the severe stretch [3]. Cells are typically considered as continuum materials and their mechanical behavior are described using continuum mechanics models. The parameters associated with models are considered to be the mechanical properties of cells which can be determined using experimental measurements. The most commonly used models are elastic and viscoelastic models based on which the elastic or dynamic modulus can be determined [4-15].

AFM is considered to be one of the best candidates for accurately measuring the load-displacement relationship of supersoft materials $(\sim \mathrm{kPa})$ at a small scale. Thus, AFM is becoming one of the most popular ways to measure the cell properties [5-18]. Although the commercial nanoindenter has been widely used to measure the mechanical properties of materials at nanoscale or microscale, the applied force range of nanoindenter is still too high to accurately measure cell properties. For example, the applied force range for the Hysitron nanoindenter is from $30 \mathrm{nN}$ to $10 \mathrm{~N}$ according to the menu [19].

The standard sharp AFM tip is likely to cause a very high stress concentration leading to highly nonlinear behavior. Also, the sharp tip may even tear the cell membrane. To avert these problems, a spherical tip is usually used instead of conventional tip in cell indentation $[7,18]$. In experiments, the radius of the indenter tip is usually chosen under $40 \mu \mathrm{m}$ [5-7,9,18], compared to cells which are typically below $40 \mu \mathrm{m}$.

\footnotetext{
*gcao2@unl.edu

†nchandra2@unl.edu
}

The cell is typically mounted on a substrate. Since the substrate is orders of magnitude stiffer than the cell by itself, the indentation force-displacement $(P-\delta)$ relationship will be influenced, especially for low cell thickness with deep indentation [5,7]. Though the substrate effect is minimal in shallow indentations, it is not practical to use shallow indentation to cells for the following reasons. In shallow indentations, it is highly difficult to accurately measure the contact area due to the surface roughness and very low stiffness of cells as well as the presence of adhesive force between the indenter tip and cell surface $[10,20,21]$. It is very important to understand and remove the substrate effect from the results of the AFM indentation measurement in order to obtain the true cell properties.

The substrate stiffening effect on the thin film indentation behavior has been widely investigated [22-24]. Based on the approaches initiated by Chen [23] and Tu et al. [24], Dimitriadis et al. introduce the correction terms into the Hertz contact model to correct the substrate stiffening effect on the elastic modulus of cell [25]. Mahaffy et al. [7] developed an analysis to obtain the viscoelastic properties of cells attached on the rigid substrate from the AFM dynamic indentation measurement. In this approach, the solution to the problem of cell indentation with substrate was assumed to be a series expansion of the standard Hertz elastic contact solution extended to linear viscoelastic materials. All of the above results and other reported cell properties measured using AFM indentation [6,9] are still based on the Hertz contact solution which is the solution derived for the semi-infinite elastic contact problem. In addition, the geometric characteristics of the cell (the cell diameter and thickness as well as the indenter tip size) are not considered, which may significantly affect the cell indentation response. For example, the substrate stiffening effect may not be just a function of cell thickness but also dependent on the indenter tip size and cell diameter. Therefore, it is highly necessary to establish an effective analysis to identify these effects and show the intrinsic properties of cell.

In this paper, the cell mechanical behavior is investigated using the semiempirical approach based on numerical simulations. In the simulations, the relationship between contact 


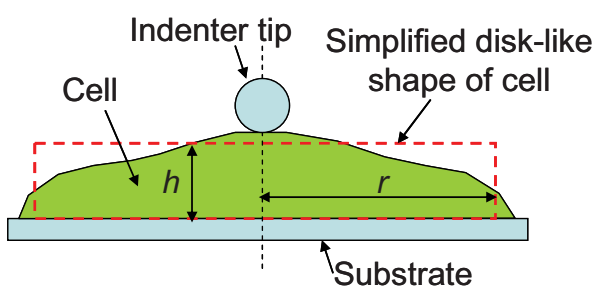

FIG. 1. (Color online) The schematic of cell indentation and simplified disklike shape.

radius and indentation displacement is established and the substrate effect is identified. The effects of indenter tip size, cell thickness, and cell radius on the indentation behavior are examined. The correcting factors are introduced into the existing indentation analysis; then cell properties can be more accurately determined. This study can help us to understand the intrinsic mechanical properties of cells and can provide a useful guideline for building the relationship between mechanical behaviors and biological functions.

\section{COMPUTATIONAL METHODS}

In the present work, the cell indentation behavior is studied using numerical simulations based on finite element modeling (FEM). After cells mounted on substrate, cells will expand themselves and the size along the normal direction of substrate is much less than the lateral size (as shown in Fig. 1). Cell geometry is typically described using two parameters: cell average radius $r$ (along the lateral direction) and thickness $h$ (along the normal direction of substrate) [4,6,10,11,26-29].

The cell is usually modeled as a continuous isotropic linear viscoelastic material. The standard linear solid (SLS) model is used to determine the dynamic modulus of cells as shown in Fig. 2. In the SLS model, the relaxation modulus is given by

$$
E(t)=E_{1}+E_{2} e^{-t / \tau},
$$

where $E_{1}$ and $E_{2}$ are elastic modulus of springs and $\tau$ is the relaxation time, $\tau=\eta / E_{2}$, where $\eta$ is the viscosity. The substrate and indenter tip are assumed to be rigid since they are usually several orders stiffer than the cell.

All FEM simulations are performed using commercial code ABAQUS v.6.8. Cells are geometrically simplified as two-dimensional (2D) axisymmetric disks. The spherical indenter tip is modeled as a 2D axisymmetric surface. The cell

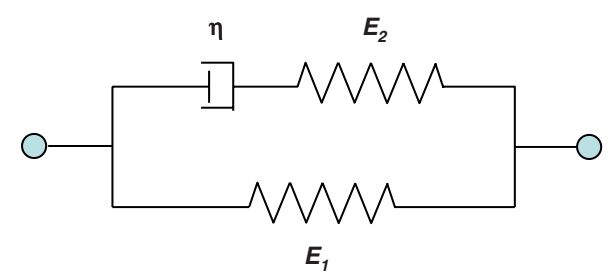

FIG. 2. (Color online) The standard linear solid model of viscoelastic material. is represented by 25000 four-node axisymmetric elements with reduced integration. The indenter tip radius $(R)$ is selected from $1 \sim 15 \mu \mathrm{m}$, which is commonly used in cell indentation $[5,7]$. The different cell radii $(r)$ are selected as $r$ $=10,20 \mu \mathrm{m}$ (typically less than $20 \mu \mathrm{m}$ in radius). The different thicknesses $(h)$ are selected as: $h=5,10 \mu \mathrm{m}$ (typically larger than $3 \mu \mathrm{m}$ ). In order to obtain an accurate contact radius, the size of surface elements in the contact area is set to less than $0.5 \%$ of the indenter tip radius. The Prony series coefficients of SLS model are $g=0.3 \sim 0.9$ and $\tau=1 \sim 10 \mathrm{~s}$ as well as the instantaneous modulus range is $E_{\text {instant }}=10$ $\sim 100 \mathrm{kPa}$ in FEM simulations. All degrees of freedom of nodes on the bottom of the cell are constrained to simulate the condition that the cell is fully adhered to the rigid substrate surface. All indentation simulations are performed based on displacement control. In order to examine the true substrate effect, the indentation displacement selected in the simulations coincide with the lowest experimentally applied value $[5,7]$.

\section{CONTACT RADIUS IN CELL INDENTATION}

In indentation tests, the accuracy of the result is strongly influenced by the accuracy demonstrated in obtaining the indentation contact radius. In the following subsections, we will discuss the effects of geometric parameters (indenter tip radius $R$, cell thickness $h$ and cell radius $r$ ) on the indentation contact radius in cell indentation and the corresponding physical mechanisms.

Based on dimensional analysis, the normalized contact radius can be described as a function of the normalized indentation displacement $(\delta / h)$, indenter tip radius $(R / h)$, cell radius $(r / R)$, and indentation force $\left(P / E h^{2}\right)$ :

$$
a / R=F\left(\delta / h, R / h, r / R, P / E h^{2}\right) .
$$

The related geometric parameters are $R, h$, and $r$. Although it is reported recently that cells might be compressible [30,31], cells are commonly assumed as incompressible $(\nu \approx 0.5)$. In the present work, the incompressible assumption is still used, and thus, $\nu$ is not considered as a variable in the present paper.

\section{A. Effects of geometric parameters on contact radius}

The variation in $a_{\text {num }} / R$ with $\delta / h$ is shown in Figs. $3(\mathrm{a})-3(\mathrm{~d})$, where $a_{\text {num }}$ is the numerical solution of the contact radius. In the figure, the indenter tip radius $R=1,15 \mu \mathrm{m}$, the cell radius $r=10,20 \mu \mathrm{m}$ and the cell thickness $h=5,10 \mu \mathrm{m}$ respectively. The FEM results are shown as thin lines (not smooth). For the sake of reference, the normalized Hertz contact radius, $a_{h} / R$, is shown as the dashed line. It is seen that when both $R$ and $h$ are small, the numerical solution $\left(a_{\text {num }}\right)$ is very close to the Hertz solution $\left(a_{h}\right)$ and $a_{\text {num }}$ is not sensitive to $r$ [the FEM result of the case with $r=10 \mu \mathrm{m}$ is overlapped with the Hertz solution in Fig. 3(a)]. With the increase in $R$ or $h, a_{\text {num }}$ deviates from $a_{h}$ and the deviation increases with $\delta / h$. When both $R$ and $h$ are large, $a_{\text {num }}$ increases with $r$. The effects of $R, h$, and $r$ on $a_{\text {num }}$ are coupled with each other. In addition, for a smaller $R$ and a larger $h$, 

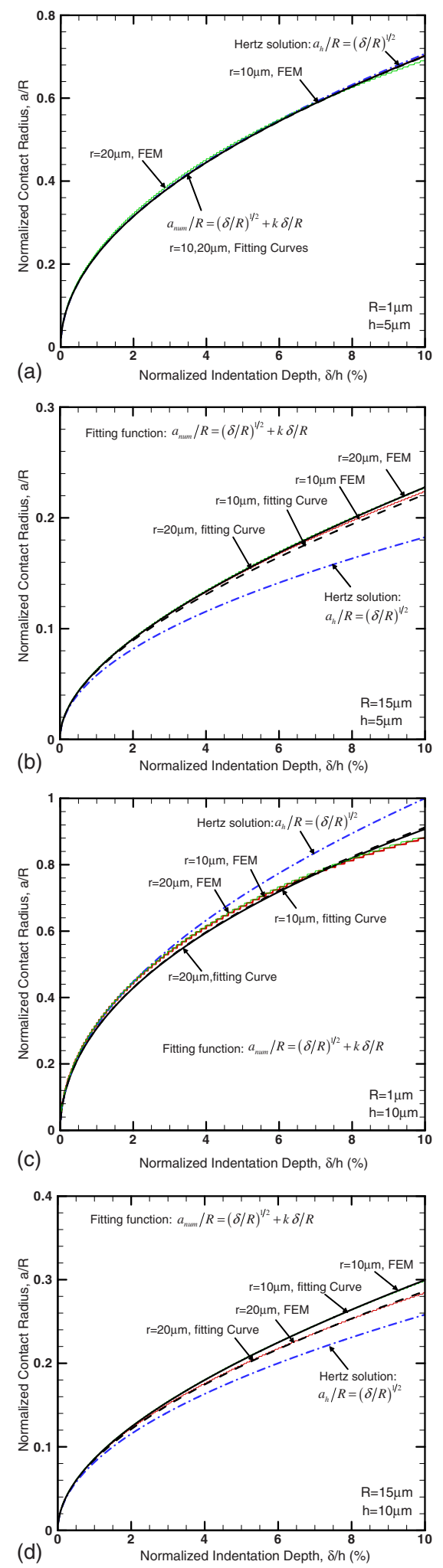

FIG. 3. (Color online) The relationship between normalized contact radius, $a / R$, and normalized indentation displacement, $\delta / h$.

$a_{\text {num }}<a_{h}$; for a larger $R$ and a smaller $h, a_{\text {num }}>a_{h}$.

For all cases in the present study $(1<R<15 \mu \mathrm{m}, h$ $=5,10 \mu \mathrm{m}, r=10,20 \mu \mathrm{m}, \delta / h<10 \%)$, the numerical solution of contact radius can be fitted as

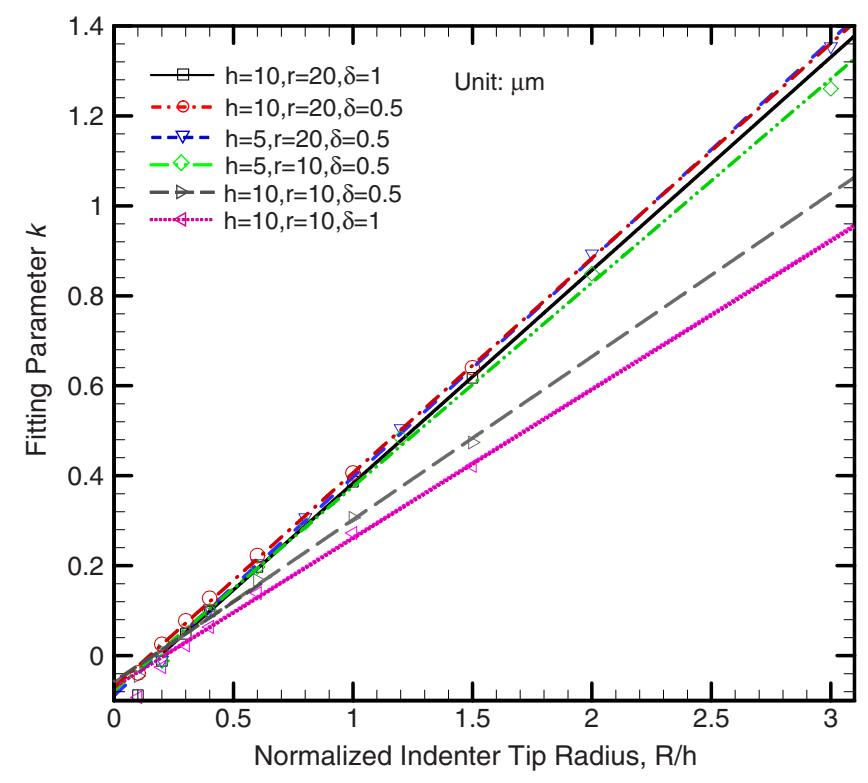

FIG. 4. (Color online) The fitting parameter $k$ in Eq. (2).

$$
\frac{a_{\text {num }}}{R}=\left(\frac{\delta}{R}\right)^{1 / 2}+k \frac{\delta}{R},
$$

where $k=k(R, h)$ is the fitting parameter, which also depends on the cell radius, $r$. The fitting curves of FEM results are shown as thick solid lines (smooth) in Figs. 3(a)-3(d). For all cases except for $R=1 \mu \mathrm{m}$ and $\delta=1 \mu \mathrm{m}, k$ can be fitted as $k=k_{1}+k_{2} R / h$, as shown in Fig. 4 . The fitting parameter, $k_{1}$, is essentially a constant and $k_{1} \approx-0.083$. The fitting parameter $k_{2} \approx 0.48$ for $r=20 \mu \mathrm{m}$. However, $k_{2}$ decreases with the increase in $\delta$ or $h$ when $r$ reduces to $10 \mu \mathrm{m}$, as shown in the Fig. 4.

\section{B. Basic mechanisms}

It is evident that all geometric parameters $R, h$, and $r$ will influence the contact radius due to nonlinear geometry, substrate, and boundary effects. Substitution of the expression of $k=k_{1}+k_{2} R / h$ into Eq. (2) leads to

$$
a_{\text {num }}=(R \delta)^{1 / 2}+k_{1} \delta+k_{2} \frac{R}{h} \delta .
$$

If $\delta \ll R$ and $\delta \ll h$, Eq. (3) converges to the Hertz solution. The second term, $k_{1} \delta$, in Eq. (3) is the geometric nonlinear correction term of the contact radius when the condition $\delta$ $\ll R$ is not satisfied. The geometric nonlinear effect increases with the increase in $\delta$ or the decrease in $R$. In the present paper, the inclusion of the first-order term of $\delta$ is accurate enough to describe the geometric nonlinear effect. In fact, for very small $R$ and large $\delta$, higher-order terms of $\delta$ are required. For example, at $R=1 \mu \mathrm{m}$ and $\delta=1 \mu \mathrm{m}$ the firstorder term of $\delta$ is not accurate enough (Fig. 4). Since $k_{1}$ is negative, the nonlinear geometric effect will reduce the contact radius compared with the Hertz solution. Generally, the nonlinear geometric effect can be reduced with a larger indenter tip size. 


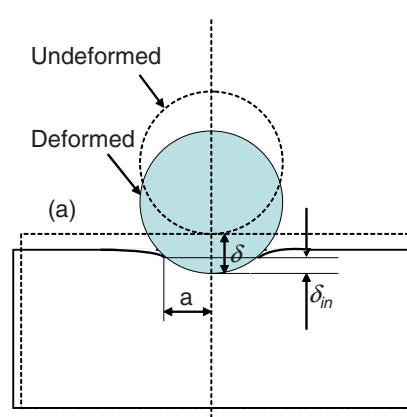

No Substrate

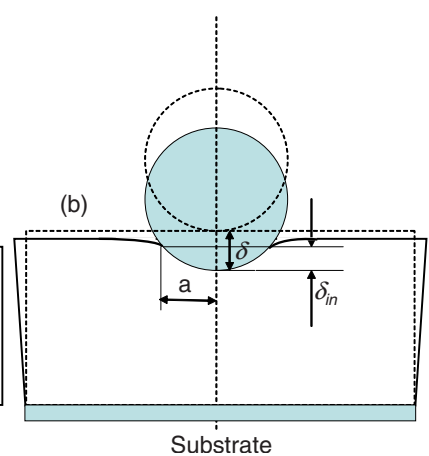

Substrate

FIG. 5. (Color online) The schematics of cell indentation with and without substrate.

The third term in Eq. (3) can be considered as the substrate effect. Figure 5 shows the schematics of cell indentation with and without substrate. The cell compression in the thickness direction (introduced by the indentation) produces the cell stretching in the radial direction due to Poisson's effect. Both the compression and the stretching cause the penetration depth, $\delta_{i n}$, to be less than the displacement of indenter tip, $\delta$. For example, $\delta \approx 2 \delta_{\text {in }}$ in the Hertz solution. If a cell is fully adhered to a substrate, the substrate will constrain the bottom surface of the cell. Due to the small cell thickness, this constraint will reduce the cell stretching in the radial direction. This constraint effect increases the penetration depth, $\delta_{i n}$, under the same applied $\delta$ compared to the case without the substrate. Thus, the contact radius will be larger than the Hertz contact radius due to the substrate effect. The substrate effect on the contact radius increases with the decrease in $h$ or increases with $R$.

The boundary effect reduces the contact radius by affecting the extent of the cell deformation along the radial direction caused by Poisson's effect. For given $R, h$, and $\delta$, a smaller cell will deform easier than a larger cell along the radial direction due to the interaction between the free surface and the indentation stress field. This can be further supported by comparing the displacements along the radial direction, $u_{11}$, of both cells. The results show that $u_{11}$ on the free surface of the smaller cell is much larger than $u_{11}$ at the same radial position of the larger cell. The easier lateral deformation will reduce the penetration depth and thus reduce the contact radius. This effect increases with $\delta$ or $R$ or decreases with the increase in $r$.

In summary, compared with the Hertz contact solution, the nonlinear geometry and the boundary effects reduce the contact radius, while the substrate effect increases the contact radius. With a large $R / h(R / h>0.2)$, the substrate effect is stronger than the nonlinear geometric and the boundary effects; and, thus, the contact radius is underestimated using Hertz contact radius. With a small $R / h(R / h<0.2)$, the substrate effect is smaller than the nonlinear geometrical effect (the boundary effect is very weak under this condition since $R / r$ is very small); and, thus, the contact radius is overestimated using Hertz contact radius. Since typically $R / h>0.2$ in the cell indentation, the contact radius is underestimated using the Hertz contact radius. This underestimation is smaller for a smaller $R / h$. In addition, the relationship between contact radius and indentation displacement is only

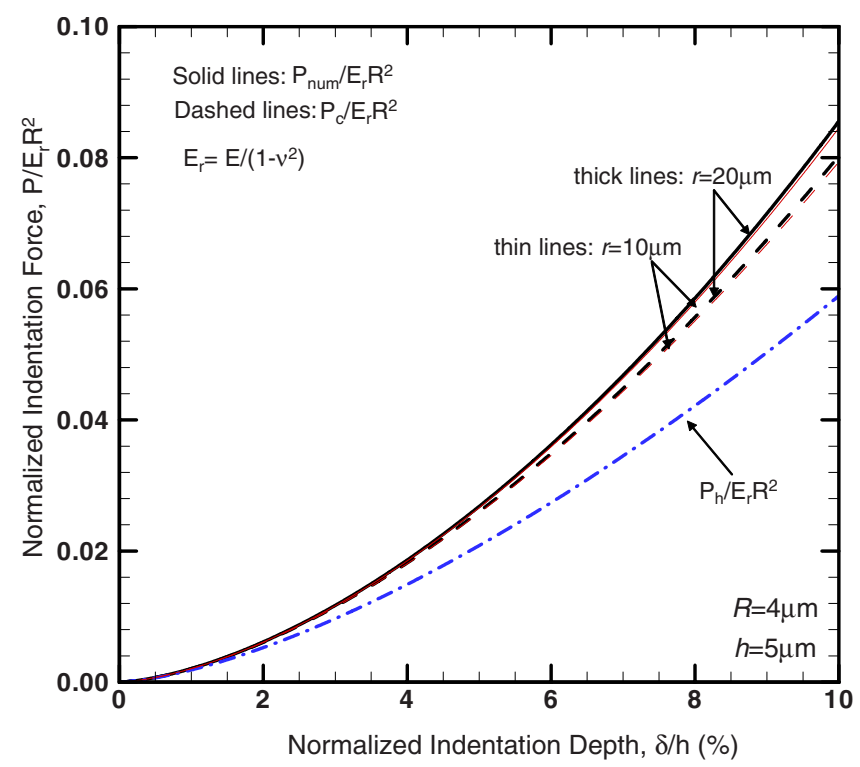

FIG. 6. (Color online) The relationship between the normalized indentation force and the normalized indentation displacement.

dependent on the geometric parameters but not the material properties of cell $(E, g$, and $\tau)$. This has been validated using FEM simulations based on the different values of $E, g$, and $\tau$ $(E=10 \sim 100 \mathrm{kPa}, g=0.3 \sim 0.9$, and $\tau=1 \sim 10 \mathrm{~s})$.

\section{EFFECT OF CONTACT RADIUS ON ELASTIC MODULUS}

Since the Hertz solution cannot accurately describe the contact radius in cell indentation, in this section, we will discuss the effect of the Hertz contact radius on determining the cell modulus based on both elastic and viscoelastic material models. In order to obtain the correct cell modulus, the correcting factor for the Hertz contact radius is required.

\section{A. Elastic materials}

For a rigid spherical indenter, the indentation force is given by [32]

$$
P=\frac{4}{3} \frac{E}{1-\nu^{2}} \frac{a^{3}}{R},
$$

where $\nu$ is the Poisson's ratio and $E$ is the elastic modulus. If the deformation is infinitesimal, $\delta \ll R$, the contact radius is commonly approximated as the Hertz solution: $a \approx a_{h}=\sqrt{R \delta}$. Thus, the indentation force can be simplified as [32]

$$
P_{h}=\frac{4}{3} \frac{E \sqrt{R} \delta^{3 / 2}}{1-\nu^{2}} .
$$

The cell elastic modulus can be directly calculated from the measured $P_{h} \sim \delta$ relationship based on Eq. (5). One example of the indentation $P \sim \delta$ curve computed from the numerical simulation is shown in Fig. 6. $P_{\text {num }}$ is the numerical solution of indentation force. The indentation force $P_{c}$ calculated from Eq. (4) based on $a_{\text {num }}$ and the Hertz solution of indentation force $P_{h}$ calculated from Eq. (5) are also indicated as a 


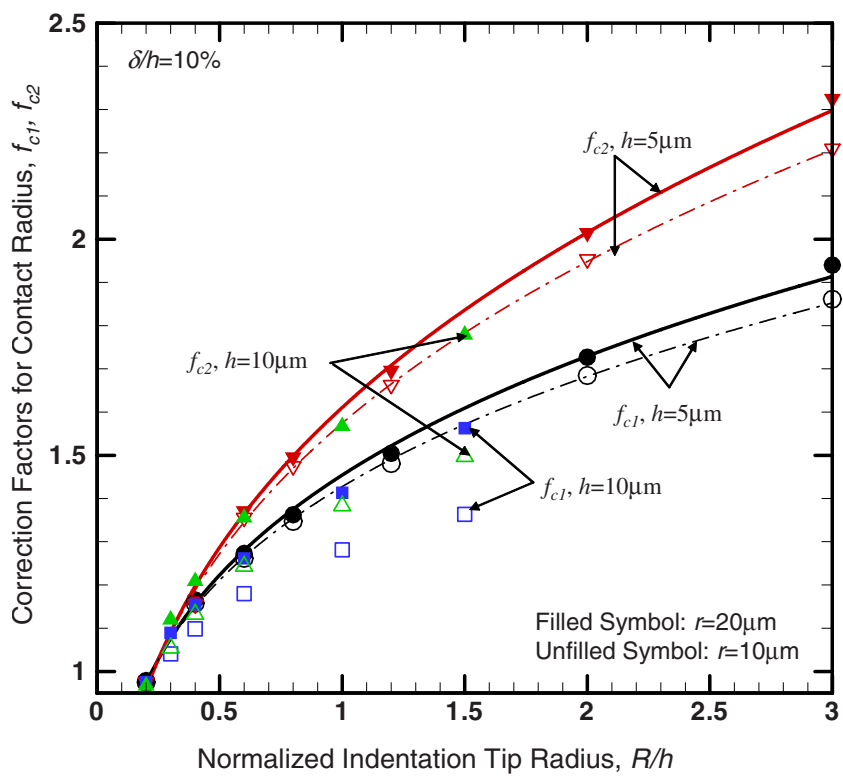

FIG. 7. (Color online) The correction factors to compensate for the effect from the Hertz contact radius on the cell elastic modulus, $f_{c 1}$ and $f_{c 2}$.

dashed line and a dash-dot line, respectively. It can be seen that $P_{\text {num }}>P_{c}>P_{h} . P_{\text {num }}>P_{h}$ even at a small $\delta$ and $P_{c}$ $\approx P_{\text {num }}$ when $\delta / h<4 \%$. With the increase in $\delta, P_{\text {num }}$ begins to deviate from $P_{c}$. This deviation is caused by the stress stiffening effect from substrate, which will be discussed in detail in the next section. The difference between $P_{c}$ and $P_{h}$ results from the inaccurate contact radius by the Hertz solution. Based on Eq. (4), the underestimation of contact radius will cause the overestimation of elastic modulus by a factor $f_{c 1}=\left(a_{\text {num }} / a_{h}\right)^{3}$ as shown in Fig. 7.

The more popular way to determine the elastic modulus in nanoindentation tests is based on the contact stiffness $d P / d \delta$, which can be calculated by the first derivative of $P$ with respect to $\delta$ in Eq. (5). The elastic modulus can be expressed as [33]

$$
\frac{E}{\left(1-\nu^{2}\right)}=\frac{d P}{d \delta} \frac{1}{2 a_{h}},
$$

where the contact stiffness $d P / d \delta$ can be measured from the $P-\delta$ curve in experiments. Since $d P / d \delta$ is easily measured in experiments (from the initial stage of the unloading curve slop), this way is more popular to measure the cell elastic modulus. However, if the contact radius is described by Eq. (2), Eq. (5) will change to

$$
P=\frac{4}{3} \frac{E}{1-\nu^{2}} \frac{(\sqrt{R \delta}+k \delta)^{3}}{R}
$$

Based on the first derivative of $P$ with respect to $\delta$ in Eq. (7), the elastic modulus is given by

$$
\frac{E}{1-\nu^{2}}=\frac{d P}{d \delta}\left(\frac{4}{3} \sum_{m=1}^{4} n_{m} \frac{m+2}{2}(k)^{m-1}(R)^{1-m / 2}(\delta)^{m / 2}\right)^{-1}
$$

where $n_{1}=1, n_{2}=3, n_{3}=3, n_{4}=1$. When $m=1$, i.e., only the first term of the sequence in Eq. (8) is considered, then it reduces to Eq. (6). Thus, if $a_{\text {num }}>a_{h}$, then Eq. (6) can overestimate the elastic modulus by a factor $f_{c 2}$ in quasistatic indentation:

$$
\begin{gathered}
f_{c 2}=\frac{2 \xi(\delta)}{3 a_{h}}, \\
\xi(\delta)=\sum_{m=1}^{4} \xi_{m}, \quad \xi_{m}=n_{m} \frac{m+2}{2}(k)^{m-1}(R)^{1-m / 2}(\delta)^{m / 2} .
\end{gathered}
$$

$f_{c 2}$ is also shown in Fig. 7. It can be seen that $f_{c 1}>f_{c 2}$ for a given $\delta$. Thus, Although Eqs. (5) and (6) are equivalent when $a \approx a_{h}=\sqrt{R \delta}$, Eq. (6) will cause a large overestimation for the elastic modulus than Eq. (5) when the contact radius is described using Eq. (2). In addition, $E$ is not a constant but a function of the indentation displacement $\delta$.

\section{B. Viscoelastic materials}

During the loading stage, the indentation force of viscoelastic material can be calculated using the correspondence principle: replacing the time-independent constant in Eq. (5) by the corresponding differential operators of the viscoelastic constitutive model [34].

$$
P(t)=\frac{4}{3\left(1-\nu^{2}\right) R} \int_{0}^{t} E(s) \frac{d\{\sqrt{R \delta(t-s)}\}^{3}}{d s} d s .
$$

In dynamic indentation with displacement control, the applied displacement profile is $\delta(t)=\delta_{0}+\Delta \delta \sin (\omega t)$. The indentation force can be also considered as a direct indentation force component superimposed with an oscillatory component $P(t)=P_{0}+\Delta P \sin (\omega t+\phi)$. The storage modulus and the loss modulus can be commonly determined based on the contact stiffness $(\Delta P / \Delta \delta)(\operatorname{Ref} .[35])$ :

$$
\begin{aligned}
& \frac{E^{\prime}}{1-\nu^{2}}=\frac{1}{2 a_{h}} \frac{\Delta P}{\Delta \delta} \cos \phi, \\
& \frac{E^{\prime \prime}}{1-\nu^{2}}=\frac{1}{2 a_{h}} \frac{\Delta P}{\Delta \delta} \sin \phi .
\end{aligned}
$$

However, when the contact radius is described by Eq. (2), based on Eq. (7), Eq. (11) will change to

$$
P(t)=\frac{4}{3\left(1-\nu^{2}\right) R} \int_{0}^{t} E(s) \frac{d\{\sqrt{R \delta(t-s)}+k \delta(t-s)\}^{3}}{d s} d s .
$$

Then, 
$P(t)=\frac{4}{3} \frac{1}{1-\nu^{2}} \sum_{m=1}^{4} n_{m}(k)^{m-1}(R)^{1-m / 2} \int_{0}^{t} E(s) \frac{d[\delta(t-s)]^{m / 2+1}}{d s} d s$

where

$$
\begin{aligned}
\delta(t)^{m / 2+1}= & \left(\delta_{0}+\Delta \delta \sin \omega t\right)^{m / 2+1} \\
= & \delta_{0}^{m / 2+1}+\frac{m+2}{2} \delta_{0}^{m / 2} \Delta \delta \sin \omega t+o(\Delta \delta), \\
& m=1 \sim 4 .
\end{aligned}
$$

Let

$$
\xi\left(\delta_{0}\right)=\sum_{m=1}^{4} n_{m} \frac{m+2}{2}(k)^{m-1}(R)^{1-m / 2}\left(\delta_{0}\right)^{m / 2} .
$$

Therefore, the oscillatory part is given by

$$
\begin{aligned}
\Delta P \sin (\omega t+\phi)= & \frac{4}{3} \frac{1}{1-\nu^{2}} \xi\left(\delta_{0}\right)\left[E^{\prime}(\omega)^{2}\right. \\
& \left.+E^{\prime \prime}(\omega)^{2}\right]^{1 / 2} \Delta \delta \sin (\omega t+\phi), \\
\tan \phi & =E^{\prime \prime}(\omega) / E^{\prime}(\omega), \\
\frac{E^{\prime}(\omega)}{1-\nu^{2}} & =\frac{3}{4} \frac{\Delta P}{\Delta \delta} \frac{1}{\xi\left(\delta_{0}\right)} \cos \phi, \\
\frac{E^{\prime \prime}(\omega)}{1-\nu^{2}} & =\frac{3}{4} \frac{\Delta P}{\Delta \delta} \frac{1}{\xi\left(\delta_{0}\right)} \sin \phi .
\end{aligned}
$$

Similar to the analysis for elastic materials, if $a>a_{h}$ $=\sqrt{R \delta}$, the conventional analysis [Eqs. (12) and (13)] will overestimate the complex modulus with the same factor:

$$
f_{c 2}=\frac{2 \xi\left(\delta_{0}\right)}{3 a_{h}\left(\delta_{0}\right)} .
$$

From Eqs. (20) and (21), the dynamic modulus components are not only functions of the frequency of the oscillatory load, $\omega$, but also functions of direct indentation displacement, $\delta_{0}$. The phase difference, $\phi$, between the indentation force and the indentation displacement is not affected by the correction of contact radius. The above results have been validated by FEM simulations with the SLS model $(g=0.3$ $\sim 0.9, \tau=1 \sim 10 \mathrm{~s}$, and $E_{\text {instant }}=10 \sim 100 \mathrm{kPa}$ ) and actually $f_{c 2}$ does not depend on the parameters in SLS model.

\section{NONLINEAR STRESS STIFFENING EFFECT}

In this section, we will discuss the substrate stiffening effect on the cell modulus based on both elastic and viscoelastic material models. This effect will cause a significant overestimation of the cell elastic or dynamic modulus based on the Hertz solution. In order to get the accurate cell moduli, the correcting factor for the substrate stiffening effect is required for the conventional analysis.

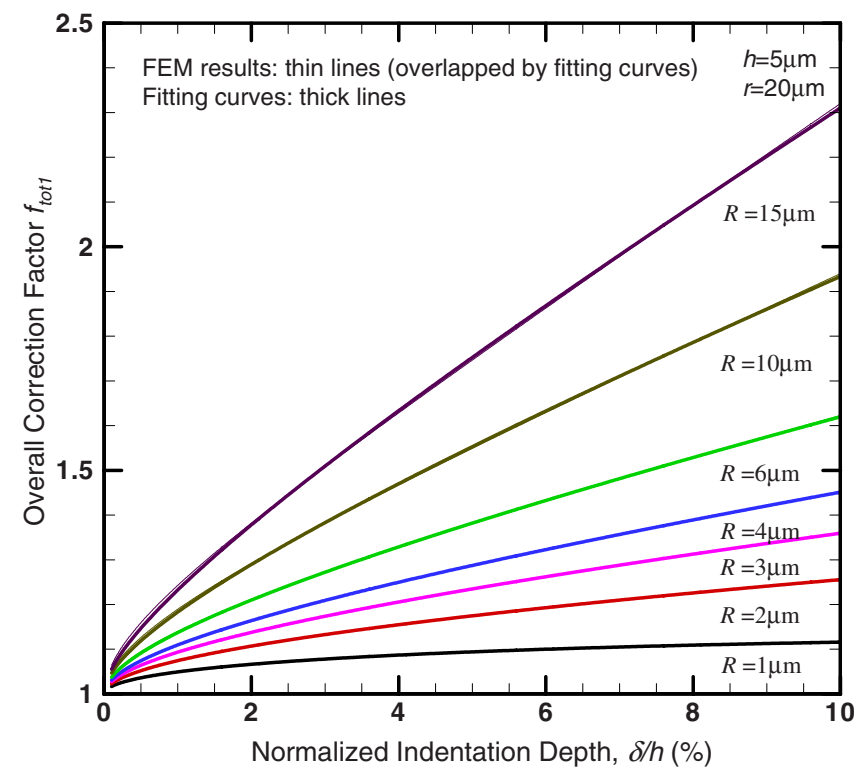

FIG. 8. (Color online) The relationship of the overall indentation correction factor $f_{\text {tot } 1}$ and normalized indentation depth, $\delta / h$.

\section{A. Elastic materials}

The overall influences of both the underestimation of contact area and the stress stiffening effect from the substrate can be simply represented by a factor $f_{\text {tot } 1}=P_{\text {num }} / P_{h}$ based on Eq. (4), which are shown as thin lines in Fig. 8. It can be seen that for all values of $R, f_{\text {tot } 1} \approx 1$ at a very small $\delta / h$; and $f_{\text {tot } 1}$ increases with $\delta / h$ and $R$. It shows that the elastic modulus is overestimated by a factor, $f_{\text {tot } 1}$, based on Eq. (4) in the quasistatic indentation. The factor includes two components:

$$
f_{t o t 1}=f_{s}+f_{c 1}-1
$$

where $f_{s}$ is from the substrate stiffening effect and $f_{c 1}$ is from the underestimation of contact radius. For example, $f_{c 1}$ $=1.92, f_{\text {tot } 1}=2.3$ at $\delta / h=10 \%$, and, thus, $f_{s}=1.38$ for the cell with $h=5 \mu \mathrm{m}, R=15 \mu \mathrm{m}$, and $r=20 \mu \mathrm{m}$. This means that the overall overestimation of the elastic modulus is $130 \%$, wherein the underestimation of the contact radius contributes to about $92 \%$, and the rest from the substrate stiffening effect is about $38 \%$.

Figure 9 shows the variation in $f_{\text {tot } 1}$ with $R / h$ at $\delta / h$ $=10 \% \cdot f_{\text {tot } 1}$ increases with $R / h$. With a larger cell radius $r$ $=20 \mu \mathrm{m}, f_{\text {tot } 1}$ is essentially insensitive to $h$. Further, with a smaller thickness $h=5 \mu \mathrm{m}, f_{\text {tot } 1}$ is insensitive to $r$; but the effect of $r$ becomes larger with $h$. The effect of $r$ on $f_{\text {tot } 1}$ arises mainly from the influence of $f_{c 1}$, which can be reduced by selecting smaller $R / h$.

For a given cell radius, $r, f_{\text {tot } 1}$ can be fitted as a function:

$$
f_{\text {tot } 1}=P_{\text {num }} / P_{h}=1+b(\delta / h)^{1 / 2}+c(\delta / h) .
$$

The fitting results of the cells with $r=20 \mu \mathrm{m}$ and $h$ $=5 \mu \mathrm{m}$ are shown as thick lines in Fig. 8 (they are overlapped with the FEM results). The coefficients $b$ and $c$ in Eq. (24) can be fitted as functions of $R / h: b=b_{1}(R / h)^{1 / 2}+b_{2}, c$ $=c_{1} R / h+c_{2}$, as shown in Fig. 10. For cells with $h=5 \mu \mathrm{m}$, 


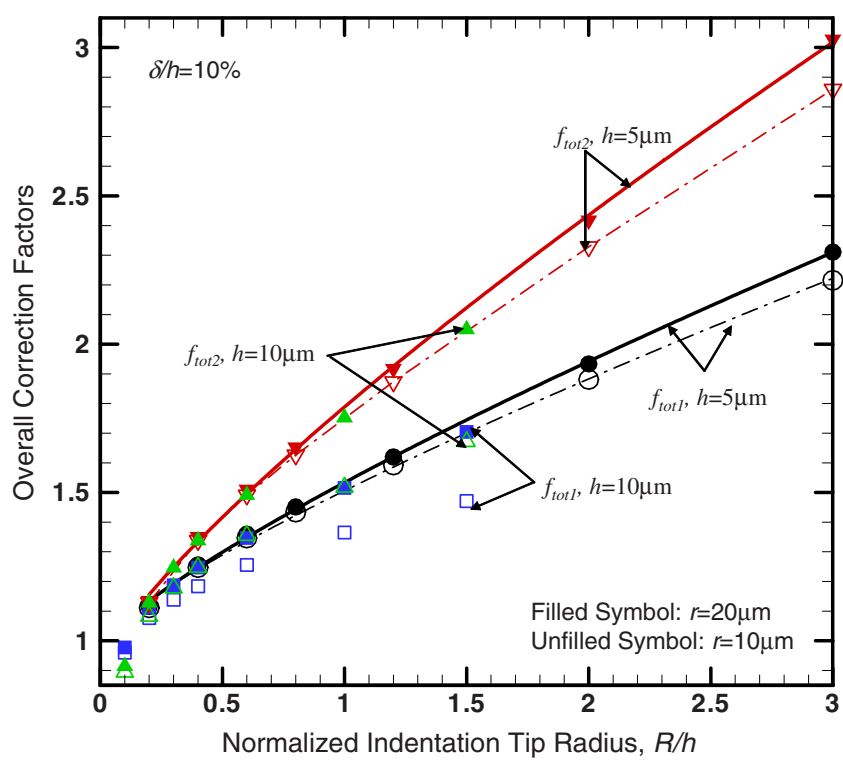

FIG. 9. (Color online) The comparison between the overall correction factors, $f_{\text {tot } 1}$ and $f_{\text {tot } 2}$ of the elastic modulus.

these constants can be found as $b_{1} \approx 0.73, b_{2} \approx 0.27$, and $c_{1}$ $\approx 3.0, c_{2} \approx-0.9$.

$P_{\text {num }}$ can be expressed as that $P_{h}$ multiplied by a function: $g(\delta / h)=1+b(\delta / h)^{1 / 2}+c(\delta / h)$, which represents the effect of geometric parameters (small $R$, large $\delta$, and low $h$ with substrate). Thus,

$$
P_{\text {num }}=\frac{4}{3} \frac{E \sqrt{R} \delta^{3 / 2}}{1-\nu^{2}} g\left(\frac{\delta}{h}\right) .
$$

Then based on the contact stiffness, $d P_{\text {num }} / d \delta$, Eq. (6) will change to

$$
\begin{gathered}
\frac{E}{1-\nu^{2}}=\frac{3}{4} \frac{d P_{n u m}}{d \delta}[\zeta(\delta)]^{-1} . \\
\zeta(\delta)=R^{1 / 2} \sum_{m=1}^{3} l_{m} \frac{m+2}{2} \delta^{m / 2} h^{(1-m) / 2},
\end{gathered}
$$

where $l_{1}=1, l_{2}=b, l_{3}=c$. Therefore, if $a>a_{h}=\sqrt{R \delta}$ and with the substrate effect, The more popular method based on the contact stiffness [Eq. (6)] will overestimate the elastic modulus by a factor $f_{\text {tot } 2}$ :

$$
f_{\text {tot } 2}=\frac{2 \zeta(\delta)}{3 a_{h}(\delta)} .
$$

It can be seen that $f_{\text {tot } 2}>f_{\text {tot } 1}$, as shown in Fig. 9. The overestimation of elastic modulus can be as high as $200 \%$ based on the more popular way [Eq. (6)], wherein the underestimation of $a$ contributes about $130 \%$, as shown in Fig. 7, and the rest arising from the stress stiffening effect.

\section{B. Viscoelastic materials}

For the viscoelastic materials with substrate effect, the components of complex modulus are given by

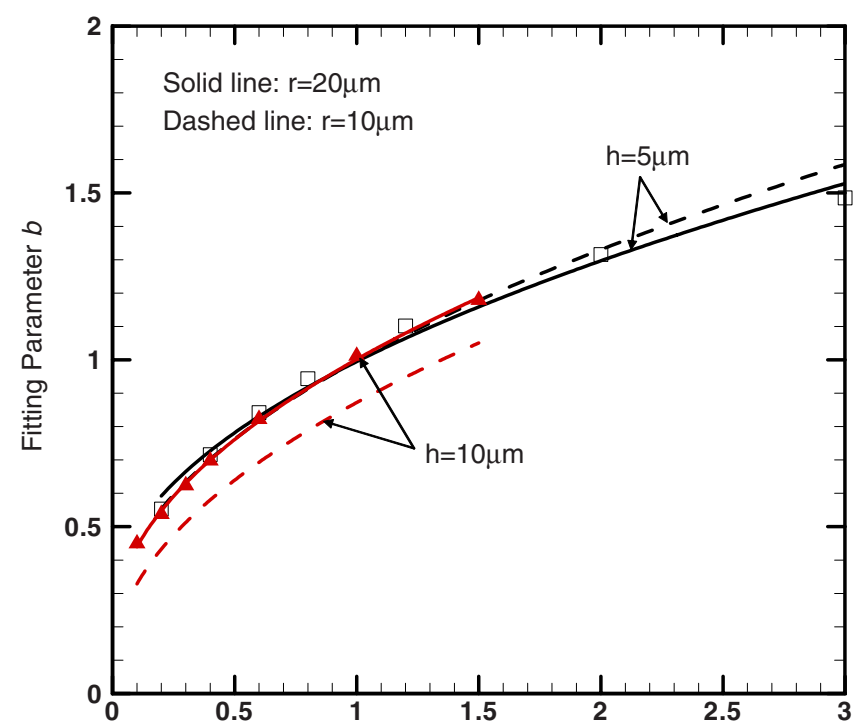

(a) The Ratio between Indenter Tip Radius and Cell Thickness, $R / h$

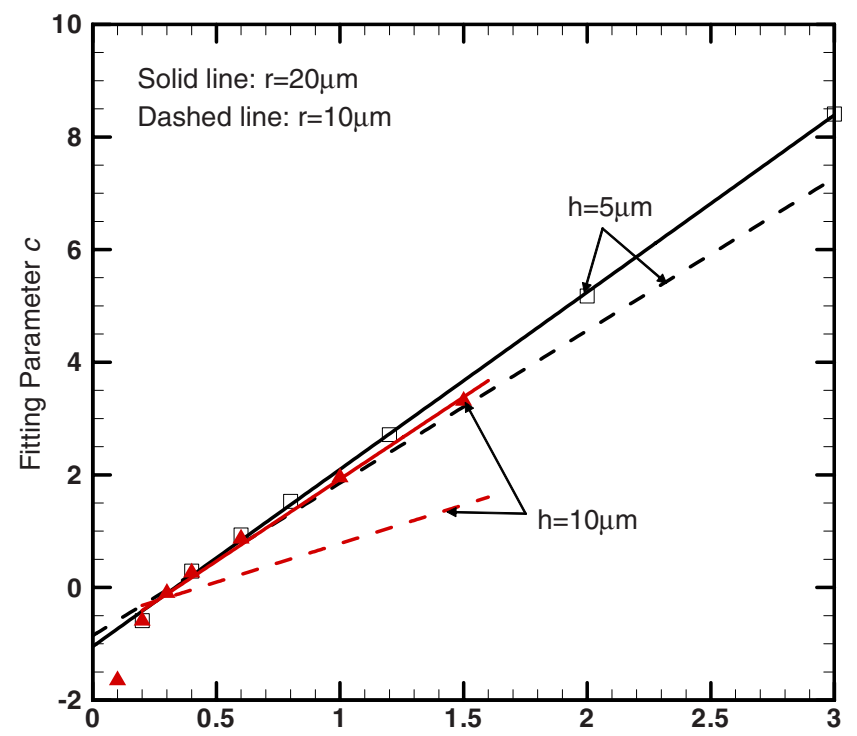

(b) The Ratio between Indenter Tip Radius and Cell Thickness, $R / h$

FIG. 10. (Color online) The fitting parameters $b$ and $c$ in Eq. (24).

$$
\begin{aligned}
& \frac{E^{\prime}(\omega)}{1-\nu^{2}}=\frac{3}{4} \frac{\Delta P}{\Delta \delta} \frac{1}{\zeta\left(\delta_{0}\right)} \cos \phi, \\
& \frac{E^{\prime}(\omega)}{1-\nu^{2}}=\frac{3}{4} \frac{\Delta P}{\Delta \delta} \frac{1}{\zeta\left(\delta_{0}\right)} \cos \phi,
\end{aligned}
$$

$$
\tan \phi=E^{\prime \prime}(\omega) / E^{\prime}(\omega),
$$

where $\zeta\left(\delta_{0}\right)=R^{1 / 2} \sum^{3} l_{m} \frac{m+2}{2} \delta_{0}^{m / 2} h^{(1-m) / 2}$. Similar to elastic ma$m=1$

terials, if $a>a_{h}=\sqrt[m=1]{R \delta}$ and with the substrate effect, Eqs. (12) and (13) will overestimate the complex modulus by the same factor: 


$$
f_{\text {tot } 2}=\frac{2 \zeta\left(\delta_{0}\right)}{3 a_{h}\left(\delta_{0}\right)} .
$$

From Eqs. (29)-(31), it can be seen that the phase difference is not affected by the substrate effect; and the components of the intrinsic dynamic modulus can be provided by Eqs. (29) and (30). The above results have been validated using FEM simulations with the SLS model $(g=0.3 \sim 0.9, \tau=1 \sim 10 \mathrm{~s}$, and $E_{\text {instant }}=10 \sim 100 \mathrm{kPa}$ ) and actually the substrate effect is not dependent on the values of $g$ and $\tau$ used in the SLS model.

\section{SUMMARY AND CONCLUSIONS}

In this work, a semiempirical analysis is built based on FEM simulations to determine the cell mechanical properties from the spherical AFM indentation response. The results show that the existing indentation analysis can overestimate the dynamic modulus by as high as $200 \%$ in some cases. The overestimation is mainly caused by the inaccurate determination of the indentation contact radius and the improper accounting of the substrate effect. The effects of inaccurate contact radius and substrate depend on the geometric parameters: indentation tip radius, cell radius, and cell thickness but do not depend on the material properties $(E, g$, and $\tau)$. Correction factors have been proposed in this work that re- late the geometric parameters to the mechanical properties in quasistatic and dynamic indentation tests.

The inaccurate determination of the contact radius is caused by the nonlinear geometric, finite boundary, and substrate effects. Substrate not only affects the value of the contact radius but also stiffens the cell. The substrate effect increases with an increase in indenter tip radius, indentation displacement, or a decrease in cell thickness. The nonlinear geometric effect increases with a decrease in indenter tip radius. The boundary effect reduces with cell thickness or/ and indenter tip radius. When the indenter tip radius is small, the nonlinear effect is dominant compared to substrate and boundary effects. When the tip radius is large, the substrate effect is dominant. To remove these effects, the correction factors $f_{t o t 1}$ and $f_{t o t 2}$ are induced, composed of the contact area correction factor and the stress stiffening factor. They increase with the increase in $R / h$ or the decrease in $h$. For a small $h$, the correction factors are not sensitive to $r$; for a large $h$, they decrease with $r$.

\section{ACKNOWLEDGMENTS}

The authors acknowledge the financial support provided by the U.S. Army Research Office for the project on "ArmyUNL Center for Trauma Mechanics," Contract No. W911NF-08-1-0483. The authors also thank Dr. Jiashi Yang and Dr. M. Negahban at UNL for many useful discussions.
[1] S. E. Cross, Y. Jin, J. Rao et al., Nat. Nanotechnol. 2, 780 (2007).

[2] Y. C. Fung, Biomechanics: Mechanical Properties of Living Tissues (Springer, New York, 1993).

[3] B. Morrison, K. E. Saatman, D. F. Meaney et al., J. Neurotrauma 15, 911 (1998).

[4] C. T. Lim, E. H. Zhou, and S. T. Quek, J. Biomech. 39, 195 (2006).

[5] Y. B. Lu, K. Franze, G. Seifert et al., Proc. Natl. Acad. Sci. U.S.A. 103, 17759 (2006)

[6] V. Lulevich, R. Zink, H.-Y. Chen et al., Langmuir 22, 8151 (2006).

[7] R. E. Mahaffy, S. Park, E. Gerde et al., Biophys. J. 86, 1777 (2004).

[8] P. Carl and H. Schillers, Pflugers Arch. Eur. J. Physiol. 457, 551 (2008).

[9] J. C. Hansen, J. Y. Lim, L.-C. Xu et al., J. Biomech. 40, 2865 (2007).

[10] S. Gupta, F. Carrillo, M. Balooch et al., J. Mater. Res. 20, 1979 (2005).

[11] T. Ohashi, Y. Ishii, Y. Ishikawa et al., Biomed. Mater. Eng. 12, 319 (2002).

[12] F. Rico, P. Roca-Cusachs, N. Gavara et al., Phys. Rev. E 72 , 021914 (2005).

[13] T. G. Kuznetsova, M. N. Starodubtseva, N. I. Yegorenkov et al., Micron 38, 824 (2007).

[14] M. Radmacher, Methods Cell Biol. 83, 347 (2007).

[15] C. Callies, P. Schöon, I. Liashkovich et al., Nanotechnology
20, 175104 (2009).

[16] E. J. Koay, A. C. Shieh, and K. A. Athanasiou, J. Biomech. Eng. 125, 334 (2003).

[17] Q. S. Li, G. Y. H. Lee, C. N. Ong et al., Biochem. Biophys. Res. Commun. 374, 609 (2008).

[18] R. E. Mahaffy, C. K. Shih, F. C. MacKintosh et al., Phys. Rev. Lett. 85, 880 (2000).

[19] http://www.hysitron.com/page_attachments/0000/0621/ TI_950_TriboIndenter_information.pdf

[20] Y. T. Cheng and C. M. Cheng, Mater. Sci. Eng. R. 44, 91 (2004).

[21] S. Gupta, F. Carrillo, C. Li et al., Mater. Lett. 61, 448 (2007).

[22] B. Oommen and K. J. Van Vliet, Thin Solid Films 513, 235 (2006).

[23] W. T. Chen, Int. J. Eng. Sci. 9, 775 (1971).

[24] Y.-O. Tu and D. C. Gazis, J. Appl. Mech. 31, 659 (1964).

[25] E. K. Dimitriadis, F. Horkay, J. Maresca et al., Biophys. J. 82, 2798 (2002).

[26] I. Kang, D. Panneerselvam, V. P. Panoskaltsis et al., Biophys. J. 94, 3273 (2008).

[27] H. Karcher, J. Lammerding, H. Huang et al., Biophys. J. 85, 3336 (2003).

[28] C. Y. Zhang and Y. W. Zhang, Philos. Mag. 87, 3415 (2007).

[29] C. Y. Zhang, Y. W. Zhang, and K. Y. Zeng, J. Mater. Res. 19, 3053 (2004).

[30] G. Ofek, D. C. Wiltz, and K. A. Athanasiou, Biophys. J. 97, 1873 (2009).

[31] W. R. Trickey, F. P. T. Baaijens, T. A. Laursen et al., J. Bio- 
mech. 39, 78 (2006).

[32] I. N. Sneddon, Int. J. Eng. Sci. 3, 47 (1965).

[33] G. M. Pharr, W. C. Oliver, and F. R. Brotzen, J. Mater. Res. 7, 613 (1992).
[34] E. H. Lee and J. R. M. Radok, ASME J. Appl. Mech. 27, 438 (1960).

[35] E. G. Herbert, W. C. Oliver, and G. M. Pharr, J. Phys. D 41, 074021 (2008). 